\title{
Outage Performance of Macrodiversity Reception in the Presence Rayleigh Short-Term Fading and Co-channel Interference
}

\author{
Branimir Jakšić, Jelena Todorović, Đoko Banđur, \\ Branko Gvozdić, Miloš Banđur
}

Faculty of Technical Sciences, University of Pristina in Kosovska Mitrovica, Knjaza Milosa 7, 38220 Kosovska Mitrovica, Serbia branimir.jaksic@pr.ac.rs, jelena.todorovic@pr.ac.rs,djoko.bandjur@pr.ac.rs, branko.gvozdic@pr.ac.rs, milos.bandjur@pr.ac.rs,

This paper presents a wireless macrodiversity communication system consisting of a receiver for macrodiversity selection combining (SC) receiver and two microdiversity SC receivers operating over correlated Gamma shadowed Rayleigh multipath fading environment in the presence of co-channel interference subject to Rayleigh short-term fading. First, we derive expression for cumulative distribution function of output signals of the both microdiversity $S C$ receivers, and then capitalizing on it, we evaluate outage probability of the macrodiversity reception. The obtained probability outage results, both numerical and those obtained by simulation, as well as, the influence of Gamma long-term severity parameter and the correlation coefficient, are graphically illustrated and discussed.

Keywords: selection combining (SC); Rayleigh fading; co-channel interference; Gamma shadowing; outage probability

\section{Introduction}

Short-term fading, long-term fading and co-channel interference degrade mobile wireless communication system performance, cause signal envelope variation and signal envelope average power variation [1]. Macrodiversity reception simultaneously reduces both long-term and short-term, fading effects on the system performance [2-5]. Microdiversity receivers mitigate short-term fading effects while macrodiversity receiver reduces long-term fading effects on wireless communications system outage performance [6-7]. Macrodiversity system is composed of a macrodiversity SC (Selection Combining) receiver and two or more microdiversity SC receivers [8-9]. Each microdiversity SC receiver selects branch with the highest signal-to-interference ratio while macrodiversity SC 
receiver selects microdiversity receiver with the highest output signal power [10$11]$.

Various system models are presented in the publicly available literature. Some of them, proven to be successful in modeling different system operating conditions, are presented in [12-17]. These models can also be applied in the modeling of wireless communication systems that use diversity technology.

The performance analysis of a SC receiver in the presence of multipath fading and co-channel interference is studied in [18-23]. A macrodiversity system composed of one macrodiversity SC receiver and two microdiversity MRC (Maximum Ratio Combining) receivers operating over Gamma shadowed Rician multipath fading channel is considered in [18]. Closed form bit error probability expression of the microdiversity SC receivers is derived, and then that expression is used for evaluation of the entire macrodiversity system bit error probability. Macrodiversity system with a macrodiversity SC receiver with L branches and MRC microdiversity receivers with $\mathrm{N}$ branches operating over Gamma shadowed Nakagami-m multipath fading channel is analyzed in [19], where the second order performance metrics such as level crossing rate and average fade duration are calculated as closed form expressions.

Performance analyzes of wireless communication systems with diversity technology and the impact of different types of fading are discussed in [6-7, 2427]. In [6], LCR (Level Crossing Rate) of the signal at the SC macrodiversity system output in the presence of $\alpha-\mu$ short-term fading and gamma long-term fading was determined. The first order statistical characteristics of an output signal of the system consisting of three MRC microdiversity receivers and one SC macrodiversity receiver were studied in [7]. Input signals of the microdiversity MRC receivers are subject of independent $\mathrm{k}-\mu$ short-term fading and correlated Gamma long-term fading. In [24], statistical moments of a signal at the output of system consisting of two EGC (Equal Gain Combining) microdiversity receivers and one SC macrodiversity receiver were determined in the presence of Nakagami-m fading. In [25], outage probability of a macrodiversity system consisting of two SC microdiversity receivers and one SC macrodiversity receiver in the presence of Fading and Weibull co-channel Interference was calculated. A study of wireless propagation in non-homogenous environment under nondeterministic LOS (Line-of-Sight) conditions, when the random nature of dominant/scattering components ratio has been considered and modeled as Gamma distribution is given in [26]. It includes closed-form expressions for PDF (Probability Density Function), CDF (Cumulative Distribution Function), outage probability and ABER (Average Bit Error Probability) of the observed fading process. In [27], expression for the chip error probability, the symbol error probability and the packet error probability are evaluated for IEEE 802.15.4 wireless channel in the presence of $\kappa-\mu$ fading, interference and additive white Gaussian noise. 
A performance analysis of SC macrodiversity reception in the presence of Rayleigh short-term fading and co-channel interference is a novelty in the publicly available literature, according to authors best knowledge. Therefore, the contribution of this paper, is as follows:

- Modeling of a wireless communication system consisting of a macrodiversity SC receiver and two microdiversity SC receivers operating over correlated Gamma shadowed Rayleigh multipath fading environment in the presence of co-channel interference subject to Rayleigh short-term fading.

- Derivation of the analytical expression for outage probability at the output of the macrodiversity system.

- Analysis of the obtained numerical results, determining the impact of system parameters on the received signal quality.

\section{System Model}

Macrodiversity system studied in this paper is composed of a macrodiversity SC receiver and two microdiversity SC receivers operating over correlated Gamma shadowed Rayleigh multipath fading channel in the presence of the co-channel interference subject to Rayleigh short-term fading. The system model is shown in Fig. 1.

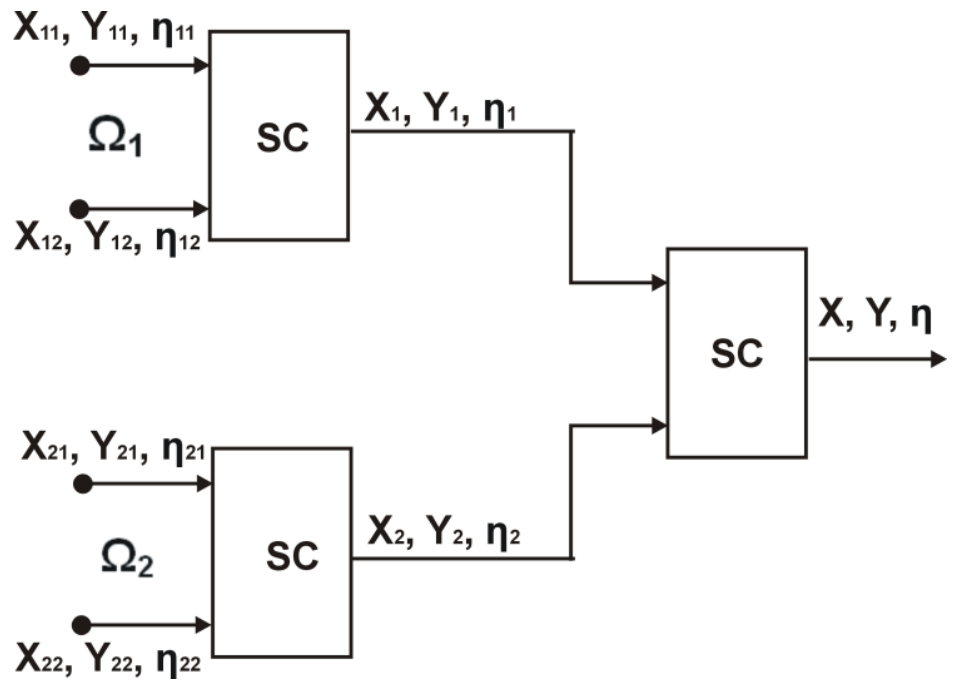

Figure 1

System model of the studied macrodiversity reception 
Received signal envelopes at the branches of the first microdiversity receiver are denoted with $x_{11}$ and $x_{12}$, and at the branches of the second microdiversity receiver with $x_{21}$ and $x_{22}$. The co-channel interference envelopes at the branches of the first microdiversity SC receiver are denoted with $y_{11}$ and $y_{12}$, and at the branches of the second microdiversity receiver with $y_{21}$ and $y_{22}$. The respective signal to interference ratios are denoted with $\eta_{11}, \eta_{12}, \eta_{21}$ and $\eta_{22}$. Desired signal envelopes at the output of the microdiversity SC receivers are denoted with $x_{1}$ and $x_{2}$, cochannel interference envelopes with $y_{1}$ and $y_{2}$, and signal to interference ratios with $\eta_{1}$ and $\eta_{2}$. Desired signal at the output of the macrodiversity SC receiver is denoted with $x$, interference with $y$, while $\eta$ is signal to interference ratio. Average power of the desired signal at the branches of the microdiversity SC receivers is denoted with $\Omega_{1}$ and $\Omega_{2}$, and the average power of the interference signal with $S_{1}$ and $S_{2}$. PDF of $x_{i j}, i=1,2 ; j=1,2$ is [2, 28]:

$$
p_{x_{i j}}=\left(x_{i j}\right)=\frac{2 x_{i j}}{\Omega_{i}} e^{-\frac{x_{i j}^{2}}{\Omega_{i}}}, \quad x_{i j} \geq 0, \quad i=1,2 ; \quad j=1,2
$$

and probability density function of $y_{i j}$ is $[2,28]$ :

$$
p_{y_{i j}}=\left(y_{i j}\right)=\frac{2 y_{i j}}{S_{i}} e^{-\frac{y_{i j}^{2}}{S_{i}}}, \quad y_{i j} \geq 0, \quad i=1,2 ; \quad j=1,2
$$

The signal to interference ratio at the branches of the microdiversity SC receivers is

$$
\eta_{i j}=\frac{x_{i j}}{y_{i j}}, \quad i=1,2 ; \quad j=1,2
$$

Probability density function of $\eta_{i j}$ is

$$
p_{\eta_{i j}}\left(\eta_{i j}\right)=\int_{0}^{\infty} d y_{i j} y_{i j} p_{x_{i j}}\left(\eta_{i j} y_{i j}\right) p_{y_{i j}}\left(y_{i j}\right)=2 \Omega_{i} S_{i} \frac{\eta_{i j}}{\left(\eta_{i j}^{2} S_{i}+\Omega_{i}\right)^{2}}, \quad i=1,2 ; \quad j=1,2(4)
$$

$\mathrm{CDF}$ of $\eta_{i j}$ is

$$
\begin{aligned}
& F_{\eta_{i j}}\left(\eta_{i j}\right)=\int_{0}^{\eta_{i j}} d t p_{\eta_{i j}}(t)=2 \Omega_{i} S_{i} \int_{0}^{\eta_{i j}} d t \frac{t}{\left(t^{2} S_{i}+\Omega_{i}\right)^{2}}=\frac{\eta_{i j}^{2}}{\eta_{i j}^{2}+\frac{\Omega_{i}}{S_{i}}}= \\
& =1-\frac{\frac{\Omega_{i}}{S_{i}}}{\eta_{i j}^{2}+\frac{\Omega_{i}}{S_{i}}}, \quad i=1,2 ; \quad j=1,2
\end{aligned}
$$


Cumulative distribution function of at outputs of microdiversity SC receivers is

$$
F_{\eta_{i}}\left(\eta_{i}\right)=F_{\eta_{i 1}}\left(\eta_{i}\right) F_{\eta_{i 2}}\left(\eta_{i}\right)=\frac{\eta_{i}^{4}}{\left(\eta_{i}^{2}+\frac{\Omega_{i}}{S_{i}}\right)^{2}}, \quad i=1,2
$$

Joint probability density function of $\Omega_{1}$ and $\Omega_{2}$ is [29]:

$$
\begin{aligned}
& p_{\Omega_{1} \Omega_{2}}\left(\Omega_{1} \Omega_{2}\right)=\frac{1}{\Gamma(c)\left(1-\rho^{2}\right) \rho^{\frac{c-1}{2}} \Omega_{0}^{c+1}} \sum_{i_{1}=0}^{\infty}\left(\frac{\rho}{\Omega_{0}\left(1-\rho^{2}\right)}\right)^{2 i_{1}+c-1} \times \\
& \times \frac{1}{i_{1} ! \Gamma\left(i_{1}+c\right)} \Omega_{1}^{i_{1}+c-1} \Omega_{2}^{i_{1}+c-1} e^{-\frac{\Omega_{1}+\Omega_{2}}{\Omega_{0}\left(1-\rho^{2}\right)}}
\end{aligned}
$$

where $c$ is Gamma fading severity parameter, $\Omega_{0}$ is the average power of $\Omega_{1}$ and $\Omega_{2}$ and $\rho$ is correlation coefficient. Joint probability density function of $\Omega_{1}$ and $\Omega_{2}$ based on (8) is plotted in Fig. 2.

The joint probability density function of $S_{1}$ and $S_{2}$ follows independent Gamma distribution:

$$
p_{S_{1} S_{2}}\left(S_{1} S_{2}\right)=\frac{1}{\Gamma(c) S_{0}^{c_{1}-1}} S_{1}^{c_{1}-1} e^{-\frac{S_{1}}{S_{0}}} \cdot \frac{1}{\Gamma(c) S_{0}^{c_{1}-1}} S_{2}^{c_{1}-1} e^{-\frac{S_{2}}{S_{0}}}
$$

where $c$ is Gamma severity parameter and $S_{0}$ is the average square value of $S_{1}$ and $S_{2}$.

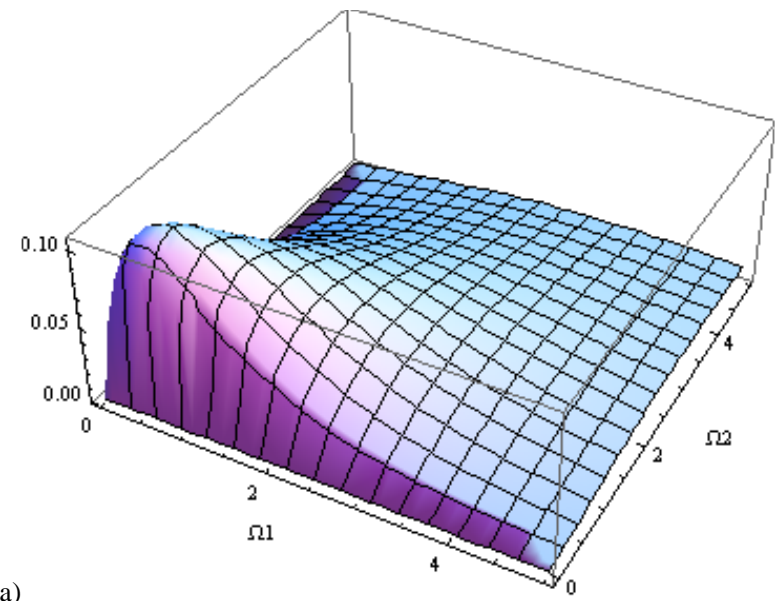




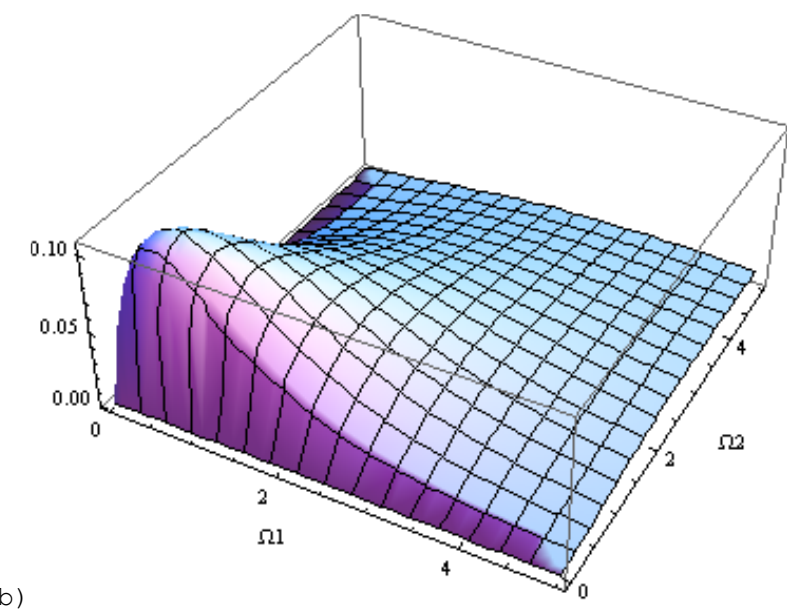

Figure 2

Joint probability density function of $\Omega_{1}$ and $\Omega_{2}$ for $\mathrm{c}=1.5$ and: a) $\rho=0.2$, b) $\rho=0.8$

\section{Analytical Results}

The algorithm for calculating the analytical expression for outage probability at the output of the macrodiversity system is presented in Fig. 3.

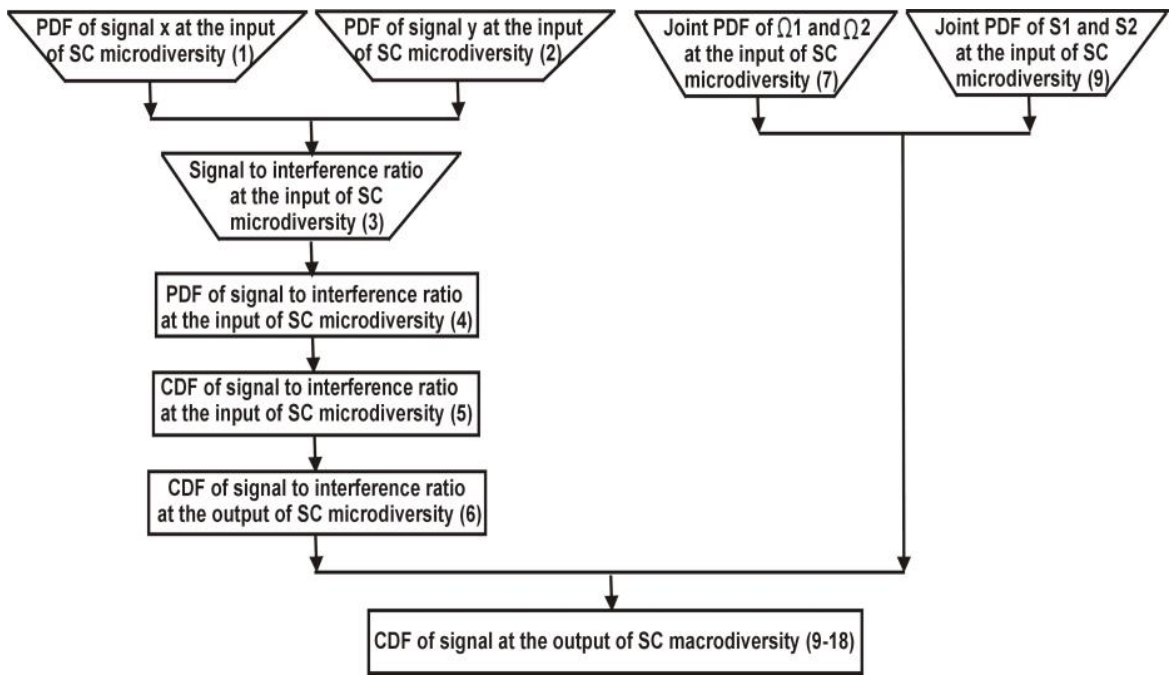

Figure 3

Algorithm for outage probability calculation 
The figure presents the necessary steps for obtaining the required expression, and based on the proposed system model given in Fig. 1. Necessary steps for obtaining the required expression are related to equations in the paper.

Macrodiversity SC receiver selects microdiversity SC receiver with the highest average power of the output signal. Therefore, cumulative distribution function of the macrodiversity SC receiver output signal is [25]:

$$
\begin{aligned}
& F_{\eta}\left(\eta / S_{1} S_{2}\right)=\int_{0}^{\infty} d \Omega_{1} \int_{0}^{\Omega_{1}} d \Omega_{2} F_{\eta_{1}}\left(\eta / \Omega_{1}, S_{1}\right) p_{\Omega_{1} \Omega_{2}}\left(\Omega_{1} \Omega_{2}\right)+ \\
& +\int_{0}^{\infty} d \Omega_{2} \int_{0}^{\Omega_{2}} d \Omega_{1} F_{\eta_{2}}\left(\eta / \Omega_{2}, S_{2}\right) p_{\Omega_{1} \Omega_{2}}\left(\Omega_{1} \Omega_{2}\right)= \\
& =F_{\eta}\left(\eta / S_{1}\right)+F_{\eta}\left(\eta / S_{2}\right)
\end{aligned}
$$

By substituting (7) and (8) into (9) and by using (5), $F_{\eta}\left(\eta / S_{1}\right)$ can be expressed as:

$$
\begin{aligned}
& F_{\eta}\left(\eta / S_{1}\right)=\int_{0}^{\infty} d \Omega_{1} \int_{0}^{\Omega_{1}} d \Omega_{2} F_{\eta_{1}}\left(\eta / \Omega_{1}, S_{1}\right) p_{\Omega_{1} \Omega_{2}}\left(\Omega_{1} \Omega_{2}\right)= \\
& =\frac{1}{\Gamma(c)\left(1-\rho^{2}\right) \rho^{\frac{c-1}{2}} \Omega_{0}^{c+1}} \sum_{i_{1}=0}^{\infty}\left(\frac{\rho}{\Omega_{0}\left(1-\rho^{2}\right)}\right)^{2 i_{1}+c-1} \frac{1}{i_{1} ! \Gamma\left(i_{1}+c\right)}\left(\Omega_{0}\left(1-\rho^{2}\right)\right)^{i_{1}+c} \times \\
& \times \frac{1}{i_{1}+c}\left(\frac{1}{\Omega_{0}\left(1-\rho^{2}\right)}\right)^{i_{1}+c} \sum_{j_{1}=0}^{\infty} \frac{1}{\left(i_{1}+c+1\right)_{\left(j_{1}\right)}}\left(\frac{1}{\Omega_{0}\left(1-\rho^{2}\right)}\right)^{j_{1}} \cdot I_{1}
\end{aligned}
$$

where $(a)_{n}$ denoting the Pochhammer symbol [30] and:

$$
\begin{aligned}
& I_{1}=\int_{0}^{\infty} d \Omega_{1} \Omega_{1}^{2 i_{1}+2 c-1+j_{1}} e^{-\frac{2 \Omega_{1}}{\Omega_{0}\left(1-\rho^{2}\right)}} \frac{1}{\left(1+\frac{\Omega_{1}}{S_{1} y^{2}}\right)^{2}}= \\
& =\left(S_{1} \eta^{2}\right)^{2 i_{1}+2 c-1+j_{1}} \int_{0}^{\infty} d y y^{2 i_{1}+2 c-1+j_{1}} e^{-\frac{2 S_{1} \eta^{2} y}{\Omega_{0}\left(1-\rho^{2}\right)}} \frac{1}{(1+y)^{2}}
\end{aligned}
$$

By using formula for confluent hypergeometric function $U(a, b, z)[30]$ :

$$
U(a, b, z)=\frac{1}{\Gamma(a)} \int_{0}^{\infty} e^{-z t} t^{a-1} \frac{1}{(1+t)^{a+1-b}} d t
$$

the integral $I_{1}$ can be written in the form: 


$$
\begin{aligned}
& I_{1}=\left(S_{1} \eta^{2}\right)^{2 i_{1}+2 c-1+j_{1}} \Gamma\left(2 i_{1}+2 c-1+j_{1}\right) \times \\
& \times U\left(2 i_{1}+2 c+j_{1}, 2 a_{1}+2 c+j_{1}-1, \frac{2 S \eta^{2}}{\Omega_{0}\left(1-\rho^{2}\right)}\right)
\end{aligned}
$$

After substitution (13) in (10), $F_{\eta}\left(\eta / S_{1}\right)$ becomes:

$$
\begin{aligned}
& F_{\eta}\left(\eta / S_{1}\right)=\frac{1}{\Gamma(c)\left(1-\rho^{2}\right) \rho^{\frac{c-1}{2}} \Omega_{0}^{c+1}} \sum_{i_{1}=0}^{\infty}\left(\frac{\rho}{\Omega_{0}\left(1-\rho^{2}\right)}\right)^{2 i_{1}+c-1} \frac{1}{i_{1} ! \Gamma\left(i_{1}+c\right)} \frac{1}{i_{1}+c} \times \\
& \times \sum_{j_{1}=0}^{\infty} \frac{1}{\left(i_{1}+c\right)} \frac{1}{\left(\Omega_{0}\left(1-\rho^{2}\right)\right)^{j_{1}}} \eta^{2\left(2 i_{1}+2 c+j_{1}\right)} \Gamma\left(2 i_{1}+2 c+j_{1}-1\right) S_{1}^{2 i_{1}+2 c+j_{1}} \times \\
& \times U\left(2 i_{1}+2 c+j_{1}, 2 a_{1}+2 c+j_{1}-1, \frac{2 S_{1} \eta^{2}}{\Omega_{0}\left(1-\rho^{2}\right)}\right)
\end{aligned}
$$

The cumulative distribution function of $\eta$ can be calculated by averaging (14), $F \eta(\eta)$ becomes:

$$
\begin{aligned}
& F_{\eta}(\eta)=\int_{0}^{\infty} d S_{1} F_{\eta}\left(\eta / S_{1}\right) p_{S_{1}}\left(S_{1}\right)= \\
& =\frac{1}{\Gamma(c)\left(1-\rho^{2}\right) \rho^{\frac{c-1}{2}} \Omega_{0}^{c+1} \Gamma(c) S_{0}^{c_{1}}} \sum_{i_{1}=0}^{\infty}\left(\frac{\rho}{\Omega_{0}\left(1-\rho^{2}\right)}\right)^{2 i_{1}+c-1} \frac{1}{i_{1} ! \Gamma\left(i_{1}+c\right)} \times \\
& \times \frac{1}{i_{1}+c} \sum_{j_{1}=0}^{\infty} \frac{1}{\left(i_{1}+c+1\right)\left(j_{1}\right)} \frac{1}{\left(\Omega_{0}\left(1-\rho^{2}\right)\right)^{j_{1}}} \eta^{2\left(2 i_{1}+2 c+j_{1}\right)} \Gamma\left(2 i_{1}+2 c+j_{1}-1\right) \cdot I_{2}
\end{aligned}
$$

where $F_{\eta}\left(\eta / S_{1}\right)$ is given by (14), $p_{S 1}\left(S_{1}\right)$ is defined over (4) and $I_{2}$ is

$$
I_{2}=\int_{0}^{\infty} d S_{2} S_{2}^{2 i_{1}+2 c+j_{1}+c_{1}-1} e^{-\frac{1}{S_{0}} S_{1}} U\left(2 i_{1}+2 c+j_{1}, 2 i_{1}+2 c+j_{1}-1, \frac{2 S_{1} \eta^{2}}{\Omega_{0}\left(1-\rho^{2}\right)}\right)
$$

By using the formula [30]:

$$
\int_{0}^{\infty} d t t^{b-1} e^{-S t} U(a, c, t)=\frac{\Gamma(b) \Gamma(b-c+1)}{\Gamma(a+b-c+1)}{ }_{2} F_{1}(b, b-c+1 ; a+b-c+1 ; 1-S)
$$


the expression for $F_{\eta}(\eta)$ becomes:

$$
\begin{aligned}
& F_{\eta}(\eta)=\frac{1}{\Gamma(c)\left(1-\rho^{2}\right) \rho^{\frac{c-1}{2}} \Omega_{0}^{c+1} \Gamma(c) S_{0}^{c_{1}}} \sum_{i_{1}=0}^{\infty}\left(\frac{\rho}{\Omega_{0}\left(1-\rho^{2}\right)}\right)^{2 i_{1}+c-1} \frac{1}{i_{1} ! \Gamma\left(i_{1}+c\right)} \times \\
& \times \frac{1}{i_{1}+c} \sum_{j_{1}=0}^{\infty} \frac{1}{\left(i_{1}+c\right)} \frac{1}{\left(\Omega_{1}\left(1-\rho^{2}\right)\right)^{j_{1}}} \eta^{2\left(2 i_{1}+2 c+j_{1}\right)} \Gamma\left(2 i_{1}+2 c+j_{1}-1\right) \times \\
& \times\left(\frac{\Omega_{0}\left(1-\rho^{2}\right)}{2 \eta^{2}}\right)^{2 i_{1}+2 c+j_{1}+c_{1}} \frac{\Gamma\left(2 i_{1}+2 c+j_{1}+c_{1}\right) \Gamma(c+2)}{\Gamma\left(2 i_{1}+3 c+j_{1}+2\right)} \times \\
& \times{ }_{2} F_{1}\left(\begin{array}{l}
2 i_{1}+2 c+j_{1}+c_{1}, c+2 ; \\
2 i_{1}+3 c+j_{1}+2 ; 1-\frac{1}{S_{0}} \frac{\Omega_{0}\left(1-\rho^{2}\right)}{2 \eta^{2}}
\end{array}\right)
\end{aligned}
$$

where ${ }_{2} F_{1}$ is hypergeometric function.

In Table 1, the number of terms to be summed in order to achieve accuracy at the desired significant digit is depicted [31] in derived expression for outage probability. As we can see from the table, how increases parameter $c$ increases the number of terms to be summed in order to achieve accuracy at the 5th significant digit. For higher values of parameter $\rho$, higher number of terms to achieve accuracy at the 5th significant digit is required.

Table 1

Terms need to be summed in the expression for cumulative distribution function to achieve accuracy at the 5th significant digit presented

\begin{tabular}{|l|l|l|l|}
\hline $\begin{array}{l}\Omega_{0}=1, \\
S_{0}=1\end{array}$ & $c=1$ & $c=2$ & $c=3$ \\
\hline$\rho=0.2$ & 58 & 51 & 44 \\
\hline$\rho=0.4$ & 60 & 51 & 44 \\
\hline$\rho=0.6$ & 66 & 56 & 48 \\
\hline$\rho=0.8$ & 78 & 62 & 56 \\
\hline
\end{tabular}

\section{Numerical Results}

Outage probability of the studied macrodiversity system, is defined as the probability that the output SIR (Signal-Interference-Ratio) falls below a given threshold $\gamma_{t h}$, and it can be expressed as $P_{\text {out }}\left(\gamma_{t h}\right)=\mathrm{F}\left(\gamma_{t h}\right)$. CDF $F\left(\gamma_{t h}\right)$ was defined by Eq. 18. Numerical results were obtained by implementing expression Eq. 18, in 
the Wolfram Mathematica software package [32]. The algorithm for calculating the CDF based on which the program code with parameter values is defined is given in Fig. 4.
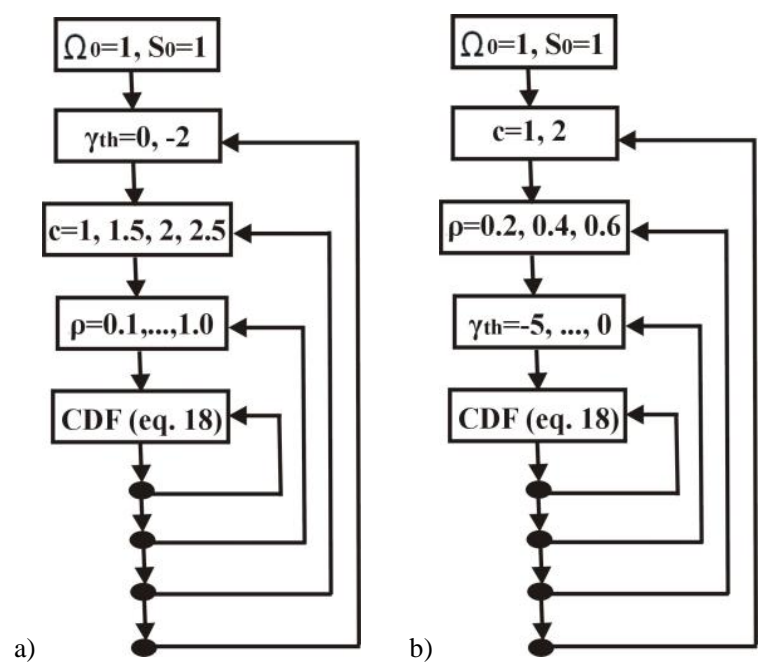

Figure 4

Algorithm for calculating the CDF of the analyzed system: a) for Figure 5, b) for Figure 6

The pseudo code for calculating the results in Fig. 5 is:

$\Omega 0=1$;

$\mathrm{S} 0=1$;

for $(\mathrm{c}=1, \mathrm{c}<=2, \mathrm{c}=\mathrm{c}+1)$ :

for $(\rho=0.2, \rho<=0.6, \rho=\rho+0.2)$ :

for $(\gamma$ th $=5, \gamma$ th $>=0, \gamma$ th $=\gamma$ th-1):

calculating Eq. (18);

The pseudo code for calculating the results in Fig. 6 is:

$\Omega 0=1$;

$\mathrm{S} 0=1$;

for $(\gamma$ th $=0, \gamma$ th $>=-2, \gamma$ th $=\gamma$ th -2$)$ :

for $(\mathrm{c}=1, \rho<=2.5, \mathrm{c}=\mathrm{c}+0.5)$ :

for $(\rho=0.1, \rho<=1.0, \rho=\rho+0.1)$ :

calculating Eq. (18); 
Outage probability of the studied macrodiversity system, and for several values of Gamma long-term fading severity parameter $c$ and Gamma long-term correlation coefficient $\rho$ is plotted in Fig. 5 and Fig. 6.

The outage probability increases as the threshold $\gamma_{t h}$ increases, and goes up to 1 for high values of $\gamma_{t h}$. When severity parameter $c$ increases, the outage probability decreases which improves system performance. The influence of the severity parameter $c$ on outage probability is higher for lower values of $\gamma_{t h}$. Values of the correlation coefficient $\rho$ can range from 0 to 1 . When the value of the correlation coefficient $\rho$ is lower, especially when it is in the range of values close to 0 , the received signals at the branches of the studied system are less correlated. It results in a higher probability of the correct signal detection, which consequently improves the system performance and makes probability outage lower. In the opposite case, for higher values of the correlation coefficient $\rho$ the outage probability is higher for the same threshold values, as it could be seen in Fig. 5. In general, influence of the correlation coefficient $\rho$ on the outage probability is higher for lower values of $\gamma_{t h}$ and moderately depends on the values of the parameter $c$, which can also be seen in Fig. 5 .

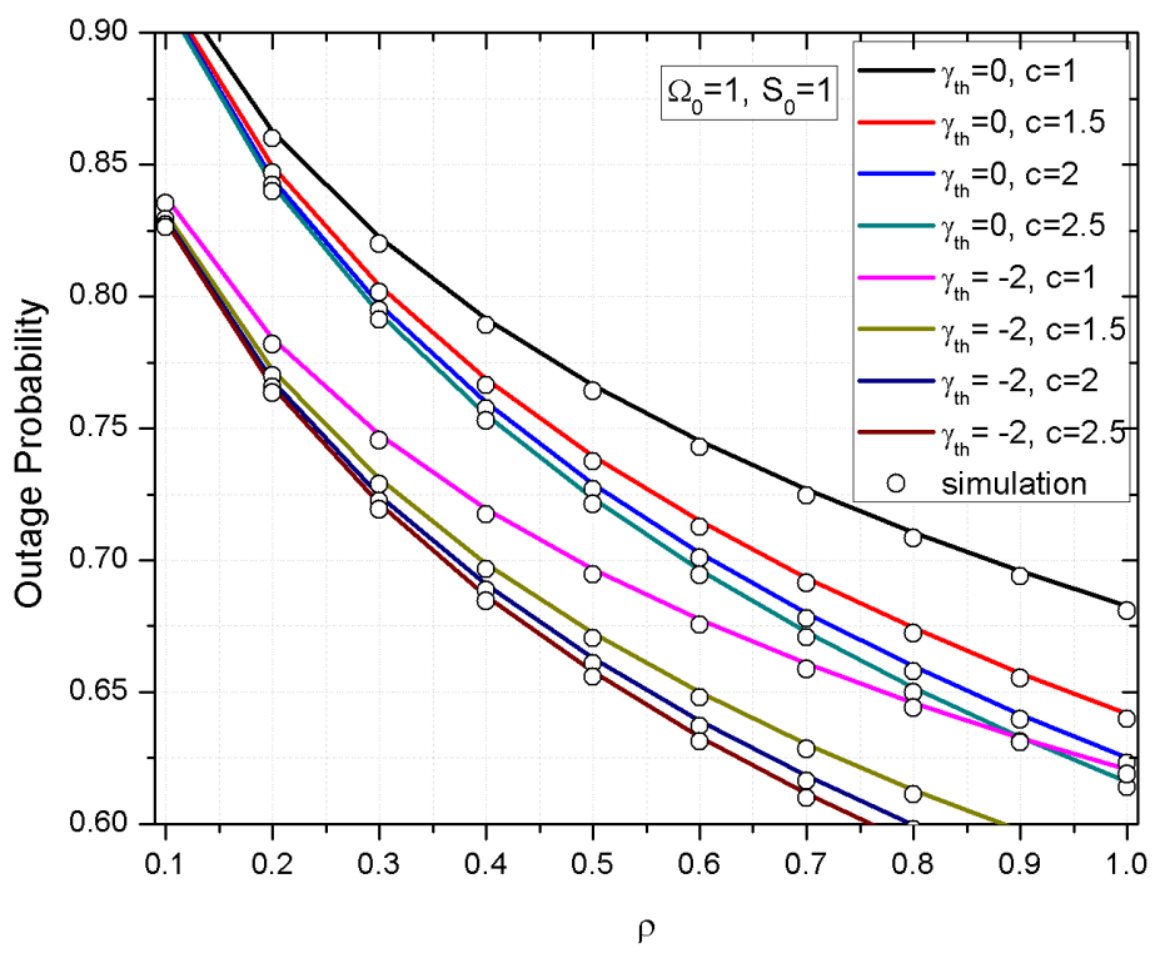

Figure 5

Outage probability versus the outage threshold for several values of $c$ and $\rho$ 
The outage probability change with correlation coefficient $\rho$ is depicted in Fig. 6 . It shows that outage probability decreases when parameter $\rho$ increases. Moreover, it is much more pronounced at the lower values of the parameter $\rho$. Also, it can be seen that for the higher values of parameter $c$ the lower outage probability values are obtained. If the values of parameter $c$ are compared, it can be seen that the difference between outage probability values is greater at lower values of parameter $c$. The behavior of the outage probability depending on the parameter $\rho$ for the various values od the parameter $c$ is identical for the various threshold values $\gamma_{t h}$, with the an exception that the lower outage probability values are being obtained for the lower threshold values $\gamma_{t h}$.

Numerical results were confirmed by Monte Carlo simulations using Matlab software package. Simulation results follow the results obtained by numerical calculation.

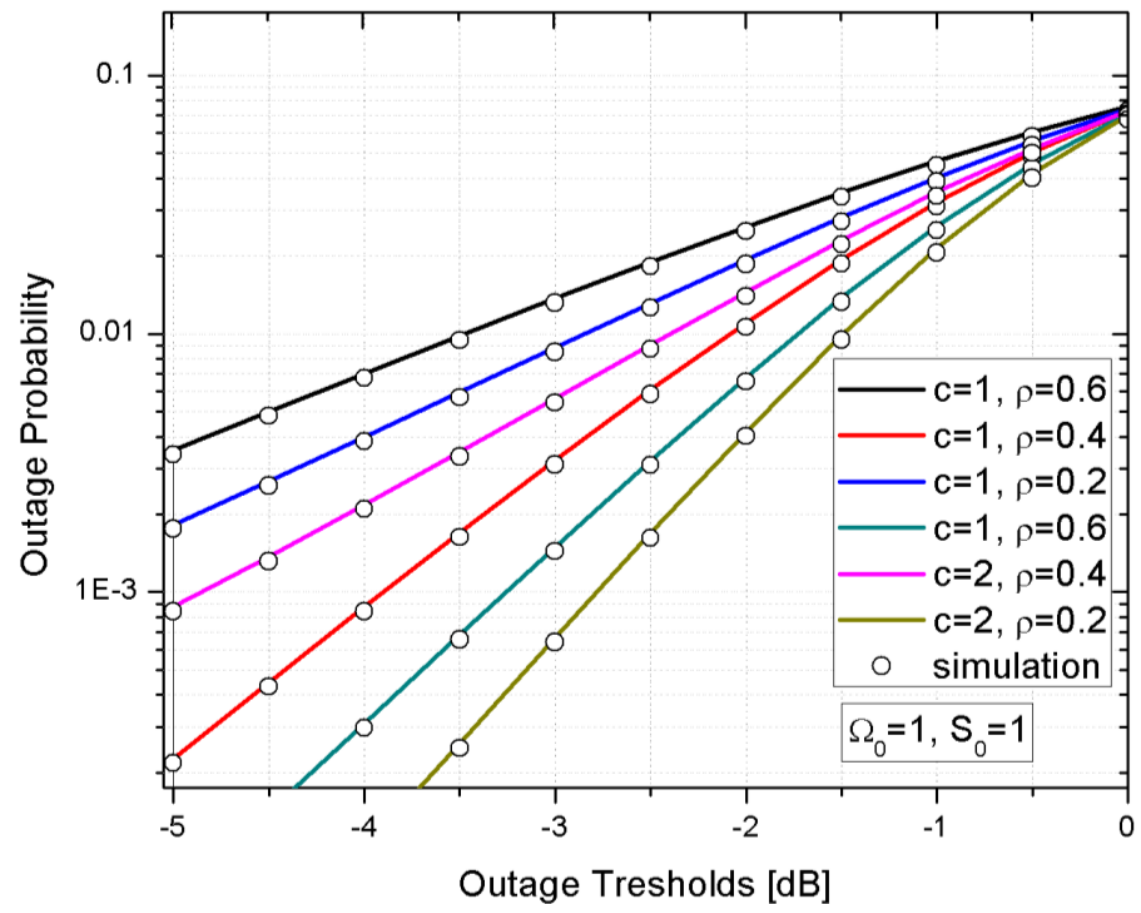

Figure 6

Outage probability versus the correlation coefficient $\rho$

\section{Conclusions}

In this paper, a macrodiversity system, composed of a macrodiversity SC receiver and two microdiversity SC receivers operating over Gamma shadowed Rayleigh multipath fading channel in the presence of Rayleigh co-channel interference, is considered. Macrodiversity receiver mitigates Gamma long-term fading effects, 
while microdiversity receivers mitigate short-term fading effects resulting in outage probability decreasing. When long-term fading severity parameter goes to infinity Gamma shadowed Rayleigh multipath channel becomes Rayleigh multipath channel, while, when Gamma long-term fading correlation coefficient goes to 1, macrodiversity system becomes microdiversity system. When correlation coefficient goes to 1, the weakest signal occurs at the both base stations, simultaneously. The closed form expression for cumulative distribution function of the microdiversity SC receiver output is derived, and then capitalizing on it, the outage probability expression of the macrodiversity SC receiver output signal is also derived. When long-term fading severity parameter goes to 1 , the expression for outage probability of the studied macrodiversity system in the presence of Gamma shadowed Rayleigh multipath fading becomes expression for outage probability of macrodiversity system in the presence of Rayleigh fading. Obtained numerical results are presented graphically to show long-term fading severity parameter and correlation coefficient influences on the considered macrodiversity system outage probability. As the Gamma severity parameter increases, outage probability decreases and as the correlation coefficient decreases, the outage probability also decreases. Outage probability increases when the threshold value increases. The influence of the Gamma severity parameter and correlation coefficient, are higher for the lower threshold values.

\section{References}

[1] S. N. Moiseev, S. A. Filin, and M. S. Kondakov: Prediction of the Standard Deviation of the Signal-to-Noise Ratio in a Data-Transmission System with Orthogonal Subcarrier Frequencies, Journal of Communications Technology and Electronics, 2008, Vol. 53, pp. 794-798, https://doi.org/10.1134/S1064226908070097

[2] S. Panic, M. Stefanovic, J. Anastasov, and P. Spalevic, Fading and Interference Mitigation in Wireless Communications, 2013, CRC Press, USA

[3] A. K. Gupta, J. G. Andrews, and R. W. Heath: Macrodiversity in Cellular Networks with Random Blockages, IEEE Transactions on Wireless Communications, 2018, Vol. 17, No. 2, pp. 996-1010, https://doi.org/10.1109/TWC.2017.2773058

[4] S. K. Yoo, S. L. Cotton, W. G. Scanlon, and G. A. Conwa: An Experimental Evaluation of Switched Combining Based Macro-Diversity for Wearable Communications Operating in an Outdoor Environment, IEEE Transactions on Wireless Communications, 2017, Vol. 16, No. 8, pp. 53385352, https://doi.org/10.1109/TWC.2017.2709298

[5] V. B. Kreindelin, D. Y. Pankratov: Analysis of the Radio Channel Capacity of a MIMO System under the Conditions of Spatially Correlated Fadings, Journal of Communications Technology and Electronics, 2019, Vol. 64, pp. 863-869, https://doi.org/10.1134/S1064226919080242 
[6] D. Krstic, B. Jaksic, M. Gligolirijevic, D. Stefanovic, and M. Stefanovic: Performance of Diversity System Output Signal in Mobile Cellular System in the Presence of $\alpha-\mu$ Short Term Fading and Gamma Long Term Fading, Radioengineering, 2016, Vol. 25, No. 4, pp. 757-762, https://doi.org/10.13164/re.2016.0757

[7] B. Jaksic, M. Stefanovic, D. Aleksic, D. Radenkovic, and S. Minic: FirstOrder Statistical Characteristics of Macrodiversity System with Three Microdiversity MRC Receivers in the Presence of $\mathrm{k}-\mu$ Short-Term Fading and Gamma Long-Term Fading, Journal of Electrical and Computer Engineering, 2016, Vol. 2016, Article ID 9689586, http://dx.doi.org/10.1155/2016/9689586

[8] G. L. Stuber: Mobile communication, 2003, Kluwer Academic Publisher, Dordrecht

[9] M. D. Yacoub: The $\eta-\mu$ distribution and the $\kappa-\mu$ distribution, IEEE Antennas and Propagation Magazine, 2007, Vol. 49, No. 1, pp. 68-81, https://doi.org/10.1109/MAP.2007.370983

[10] G. Malmgre: On the Performance of Single Frequency Networks in Correlated Shadow Fading, IEEE Transactions on Broadcasting, 1997, Vol. 43, No. 2, pp. 155-165, https://doi.org/10.1109/11.598364

[11] H. Surawereea, R. Luie, Y. Karagiannidis, G. Li, and B. Vucetic: Two Hop Amplify-and-Forward Transmission in Mixed Rayleigh and Rician Fading Channels, IEEE Communications Letters, 2009, Vol. 13, No. 4, pp. 227 229, https://doi.org/10.1109/LCOMM.2009.081943

[12] C.-F. Juang, Y.-Y. Lin, and R.-B. Huang: Dynamic system modeling using a recurrent interval-valued fuzzy neural network and its hardware implementation, Fuzzy Sets and Systems, 2011, Vol. 179, Iss. 1, pp. 83-99, https://doi.org/10.1016/j.fss.2011.05.015

[13] R. Precup, T. Teban, A. Albu, A. Borlea, I. A. Zamfirache, and E. M. Petriu: Evolving Fuzzy Models for Prosthetic Hand Myoelectric-Based Control, IEEE Transactions on Instrumentation and Measurement, 2020, Vol. 69, No. 7, pp. 4625-4636, https://doi.org/10.1109/tim.2020.2983531

[14] C. Pozna, and R.-E. Precup: Applications of Signatures to Expert Systems Modelling, Acta Polytechnica Hungarica, 2014, Vol. 11, No. 2, pp. 21-39, https://doi.org/10.12700/APH.11.02.2014.02.2

[15] R. Zall, and M. R. Kangavari: On the Construction of Multi-Relational Classifier Based on Canonical Correlation Analysis, International Journal of Artificial Intelligence, 2019, Vol. 17, No. 2, pp. 23-43

[16] E.-L. Hedrea, R.-E. Precup, R.-C. Roman, and E. M. Petriu: Tensor product-based model transformation approach to tower crane systems modeling, Asian Journal of Control, 2021, pp. 1-11, https://doi.org/10.1002/asjc.2494 
[17] W. Cheng, and C. Juang: A Fuzzy Model With Online Incremental SVM and Margin-Selective Gradient Descent Learning for Classification Problems, IEEE Transactions on Fuzzy Systems, 2014, Vol. 22, No. 2, pp. 324-337, https://doi.org/10.1109/tfuzz.2013.2254492

[18] V. Milenkovic, N. Sekulovic, M. Stefanovic, and M. Petrovic: Effect of Microdiversity and Macrodiversity on Average Bit Error Probability in Gamma Shafowed Rician Fading Channels, ETRI Journal, 2010, Vol. 32, No. 3, pp. 463-467, https://doi.org/10.4218/etrij.10.0209.0448

[19] N. Sekulovic, M. Stefanovic, D. Milovic, and S. Stanojcic: Second-Order Statistics of System with N-branch Microdiversity and L-branch Macrodiversity Operating over Gamma Shadowed Nakagami-m Fading Channels, International Journal of Communication Systems, 2014, Vol. 27, No. 2, pp. 390-400, https://doi.org/10.1002/dac.2369

[20] M. C. Stefanovic, D. L. Draca, A. S. Panajotovic, and N. M Sekulovic: Performance Analysis of System with L-branch Selection Combining over Correlated Wibull Fading Channels in the Presence of Co-channel Interference, International Journal of Communication Systems, 2010, Vol. 23, No. 2, pp. 139-150, https://doi.org/10.1002/dac.1050

[21] M. C. Ju, and K. S. Hwang: Outage Equivalence of Opportunistic Relaying and Selection Cooperation in Presence of Co-Channel Interference, IEEE Transactions on Wireless Communications, 2015, Vol. 14, No. 6, 29812991, https://doi.org/10.1109/TWC.2015.2398871

[22] B. Bhargav, C. R. N. da Silva, Y. J. Chun, S. L. Cotton, and M. D. Yacoub: Co-Channel Interference and Background Noise in $\kappa-\mu$ Fading Channels, IEEE Communications Letters, 2017, Vol. 21, No. 5, pp. 1215-1218, https://doi.org/10.1109/LCOMM.2017.2664806

[23] M. D. Yacoub: The $\alpha-\eta-\kappa-\mu$ Fading Model, IEEE Transactions on Antennas and Propagation, Vol. 64, No. 8, pp. 3597-3610, https://doi.org/10.1109/TAP.2016.2570235

[24] N. Djordjević, B. Jakšić, A. Matović, M. Matović, and M. Smilić: Moments of Microdiversity EGC receivers and Macrodiversity SC Receiver Output Signal over Gamma Shadowed Nakagami-m Multipath Fading Channel, Journal of Electrical Engineering - Elektrotechnický časopis, 2015, Vol. 66, No. 6, pp. 348-351, https://doi.org/10.2478/jee-2015-0058

[25] M. Perić, B. Jakšić, D. Aleksić, D. Randjelović, and M. Stefanović: Outage Probability of Macrodiversity Reception in the Presence Fading and Weibull Co-Channel Interference, Tehnički vjesnik - Technical Gazette, 2018, Vol. 25, No. 2, pp. 376-381, https://doi.org/10.17559/TV20161227102847

[26] D. Bandjur, B. Jaksic, S. Panic, M. Bandjur, A. Matovic, and E. Mekic: Transmission over kappa-mu Fading channels with Gamma distributed 
random Line-of-sight components, Revue Roumaine des Sciences Techniques - Série Électrotechniqueet Énergétique, 2017, Vol. 62, No. 2, pp. 179-184

[27] Đ. Banđur, B. Jakšić, A. Raičević, B. Popović, and M. Banđur: Performance Analysis of an IEEE 802.15.4 Network Operating Under $\kappa-\mu$ Fading, Interference and AWGN, Iranian Journal of Science and Technology, Transactions of Electrical Engineering, 2020, https://doi.org/10.1007/s40998-020-00329-1

[28] Y. Chen and C. Tellambura: Performance Analysis of Three-Branch Selection Combining over Arbitrarily Correlated Rayleigh-Fading Channels, IEEE Transactions on Wireless Communications, 2005, Vol. 4, No. 3, pp. 861-865, https://doi.org/10.1109/TWC.2005.847109

[29] B.Jaksic, D. Stefanovic, M. Stefanovic, P. Spalevic, and V. Milenkovic: Level Crossing Rate of Macrodiversity System in the Presence of Multipath Fading and Shadowing, Radioengineering, 2015, Vol. 24, No. 1, pp. 185191, https://doi.org/10.13164/re.2015.0185

[30] I. S. Gradshteyn, and I. M. Ryzhik: Table of Integrals, Series and Products, 2000, Academic Press, San Diego, USA

[31] B. S. Jakšić: Level Crossing Rate of Macrodiversity SC Receiver with two Microdiversity SC Receivers over Gamma Shadowed Multipath Fading Channel, Facta Universitatis, Ser. Autom. Control Robot., 2015, Vol. 14, No. 2, pp. 87-98

[32] Wolfram Mathematica: https://www.wolfram.com/ 